\title{
Radiofrequency Thermal Ablation versus Bipolar Electrocautery for the Treatment of Inferior Turbinate Hypertrophy: Comparison of Efficacy and Postoperative Morbidity
}

\author{
Sinan Uluyol ${ }^{1}$ Nermin Erdas Karakaya ${ }^{1}$ Mehmet Hafit Gur ${ }^{1}$ Saffet Kilicaslan ${ }^{1}$ \\ Esin Ozlem Kantarcioglu ${ }^{1}$ Ozlem Yagiz ${ }^{2}$ Ilker Burak Arslan ${ }^{2}$
}

${ }^{1}$ Department of Otolaryngology, Van Region Training and Research Hospital, Van, Turkey

2 Department of Otolaryngology, Tepecik Training and Research Hospital, Izmir, Turkey

Int Arch Otorhinolaryngol 2016;20:2-5.
Address for correspondence Sinan Uluyol, MD, Department of Otolaryngology Van Training and Research Hospital, Van/Edremit, Van 65300, Turkey (e-mail: sinanuluyol@hotmail.com).

\begin{abstract}
Introduction Numerous surgical methods are used to treat nasal obstruction due to inferior turbinate hypertrophy. The primary goal of the therapy is to maximize the nasal airway for as extended a period of time as possible while minimizing therapeutic complications.

Objectives The aim of this study was to assess the effects of radiofrequency thermal ablation (RFTA) and bipolar electrocautery (BEC) on the removal of nasal obstruction in patients with inferior turbinate hypertrophy and on nasal mucociliary clearance (MCC). Patients in both groups were also evaluated in terms of postoperative morbidity.

Methods We compared the outcomes of two groups of patients: those treated with RFTA $(n=23)$ and those who underwent BEC $(n=20)$. Nasal obstruction was graded using a visual analog scale (VAS) and MCC was measured using a saccharin clearance test. Both measurements were performed before and 2 months after treatment.

Results Pre- and postoperative VAS scores showed significant improvement for both groups. However, MCC results did not significantly differ between two groups. Neither

Keywords

- mucociliary clearance

- nasal obstruction

- turbinates edema nor crust formation persisted for more than 1 week in any patients.

Conclusion Submucosal cauterization with preservation of the nasal mucosa and periosteum is as effective and safe as RFTA and should be considered when planning inferior turbinate interventions.
\end{abstract}

\section{Introduction}

Nasal obstruction is one of the most common symptoms encountered by otorhinolaryngologists in clinical practice. It is usually caused by hypertrophy of the inferior turbinate. Among the surgical methods commonly used to treat nasal obstruction due to turbinate hypertrophy are cryosurgery, electrocautery, partial turbinectomy, laser turbinoplasty, and radiofrequency thermal ablation (RFTA). ${ }^{1}$

The primary goal of therapy is to maximize the nasal airway for as extended a period of time as possible while minimizing therapeutic complications, such as crust formation, nasal drying, hemorrhage, and edema. ${ }^{2}$ received

February 9, 2015

accepted

March 25, 2015

published online

April 30, 2015
DOI http://dx.doi.org/

10.1055/s-0035-1551553. ISSN $1809-9777$.
Copyright $(2016$ by Thieme Publicações License terms

Ltda, Rio de Janeiro, Brazil
(ब) $\Theta \circledast$ 
Preserving mucosal surfaces and nasal epithelial function during reduction of the hypertrophied inferior turbinate is important for mucociliary clearance (MCC) because MCC is a primary defense mechanism in the upper respiratory system. $^{3}$ MCC can be measured by various techniques. The saccharin test is a simple test used to evaluate MCC. In the presence of normal mucociliary action, the saccharin is swept backward to the nasopharynx and a sweet taste is perceived by the patient. 4

The aim of this study was to assess the efficacy of RFTA and bipolar electrocautery (BEC). Patients with inferior turbinate hypertrophy treated with either of these procedures were also evaluated in terms of postoperative morbidity. The advantages and disadvantages of each treatment were discussed.

\section{Patients and Methods}

From October 2014 to December 2014, 23 patients (10 women, 13 men) who underwent RFTA and 20 patients (11 women, 9 men) who underwent BEC were recruited. Inclusion criteria for both groups were nasal obstruction caused only by inferior turbinate hypertrophy and age over 18 years. Patients with deviation of the nasal septum or diseases such as allergic rhinitis and sinonasal infections were excluded, as were smokers. Subjective assessments of nasal patency, MCC, and postoperative morbidity (edema, nasal bleeding, and crusting) were determined using a visual analog scale (VAS), the saccharin clearance test (SCT), and endoscopic examination, respectively. This study was performed in accordance with the Helsinki Declaration of the World Medical Association and was approved by the Research Ethics Committee of a tertiary referral center. Surgeries were performed under local anesthesia.

For RFTA, the probe was placed longitudinally and submucosally in the anterior, middle, and posterior portions of the turbinate. The procedure was completed by applying $350 \mathrm{~J}$ of energy for 20 seconds in each of the three areas. For BEC, a bipolar probe delivering $20 \mathrm{~W}$ of energy was inserted between the submucosa and periosteum in the anterior, middle, and posterior portions of the turbinate. Bipolar cauterization was stopped when minimal coagulation of the nasal mucosa was achieved, as determined visually by whitening of the treated tissues. The conchal and periosteal mucosae were preserved.

Patients in the RFTA and BEC groups underwent VAS evaluation and an SCT before and 2 months after the respective procedure. SCT time was measured by placing a fourth of a saccharin tablet (5 $\mathrm{mg}$ saccharin) behind the anterior edge of the inferior turbinate. The interval between the beginning of the test and the point at which the taste was perceived by the patient was recorded in seconds. In the VAS evaluation of nasal patency, a score of 0 indicated no nasal obstruction and 10 complete nasal obstruction. Edema, nasal bleeding, and crusting were assessed via nasal endoscopic examination performed postoperatively during routine weekly visits. The date when each sign disappeared was recorded. Statistical analysis was performed using the Statistical Package for Social Sciences, version 20.0 for Windows software (SPSS Inc., Chicago, Illinois, United States). A two-tailed $t$ test was used for descriptive statistical analysis (mean \pm standard deviation) of quantitative data. A $p$ value $<0.05$ was considered to indicate statistical significance.

\section{Results}

The mean age of the patients was $32.08 \pm 7.42$ years in the RFTA group and $29.66 \pm 9.33$ years in the BEC group. In the RFTA group, the mean preoperative MCC was $561.3 \pm 69$ second (range: 465 to 713 seconds and the VAS score for nasal patency was $7.13 \pm 0.9$. Postoperatively, the corresponding values were $560.8 \pm 64$ seconds (range: 450 to 725 seconds and $3.56 \pm 0.8$ ). The pre- and postoperative VAS scores, but not the MCC values, were significantly different $(p=0.001$ and $p=0.86$, respectively). In the BEC group, the mean MCC was $570.6 \pm 61$ seconds (range: 435 to 735 seconds before treatment and $584.9 \pm 61$ seconds (range: 460 to 690 seconds 2 months after treatment, and the difference between two tests was not statistically significant $(p=0.75)$. Mean VAS score for nasal patency was $7.1 \pm 1.13$ before treatment and $3.4 \pm 1.02$ months after treatment, and the difference between the two results was statistically significant $(p=0.001)$. - Tables 1 and 2 present the SCT and VAS data for the RFTA and BEC groups. No bleeding was observed in either group. Edema and crusting in the turbinate disappeared in all patients by the end of the first week. - Figs. 1 and 2 show the turbinates pre and postoperatively for both techniques.

\section{Discussion}

Reduction of the volume of the inferior turbinate can be achieved by a variety of methods. Turbinate reduction procedures should cause minimal patient discomfort and few or no adverse effects while preserving the physiologic function of the turbinate, such as MCC. ${ }^{2}$ Surgical treatments of the inferior turbinate may lead to deterioration of MCC by damaging the nasal mucosa. The MCC system is important

Table 1 Saccharin clearance test results

\begin{tabular}{|l|l|l|l|}
\hline & Preoperative & Postoperative & $\boldsymbol{p}^{\mathbf{a}}$ \\
\hline Radiofrequency thermal ablation (s) & $561.3 \pm 69$ & $560.8 \pm 64$ & 0.86 \\
\hline Bipolar electrocautery (s) & $570.6 \pm 61$ & $584.9 \pm 61$ & 0.25 \\
\hline
\end{tabular}

${ }^{\mathrm{a}}$ Two-tailed $t$ test. 
Table 2 Visual analog score results

\begin{tabular}{|l|l|l|l|}
\hline & Preoperative & Postoperative & $p^{\mathbf{a}}$ \\
\hline Radiofrequency thermal ablation & $7.13 \pm 0.9$ & $3.56 \pm 0.8$ & 0.001 \\
\hline Bipolar electrocautery & $7.1 \pm 1.13$ & $3.44 \pm 1$ & 0.001 \\
\hline
\end{tabular}

${ }^{\text {a } T w o-t a i l e d ~} t$ test.
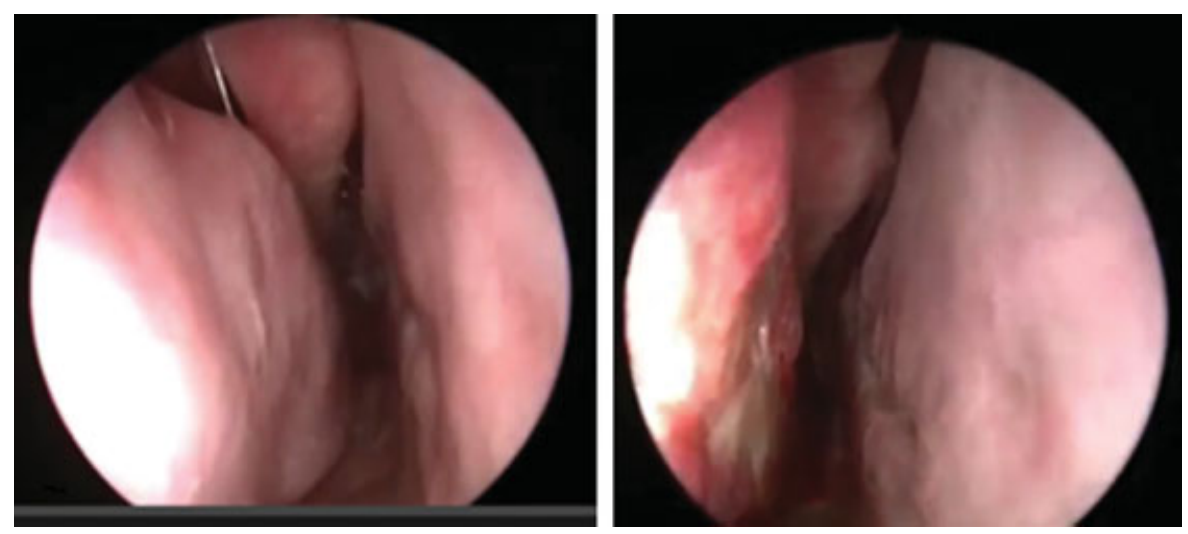

Fig. 1 Right inferior turbinates before and after radiofrequency thermal ablation.
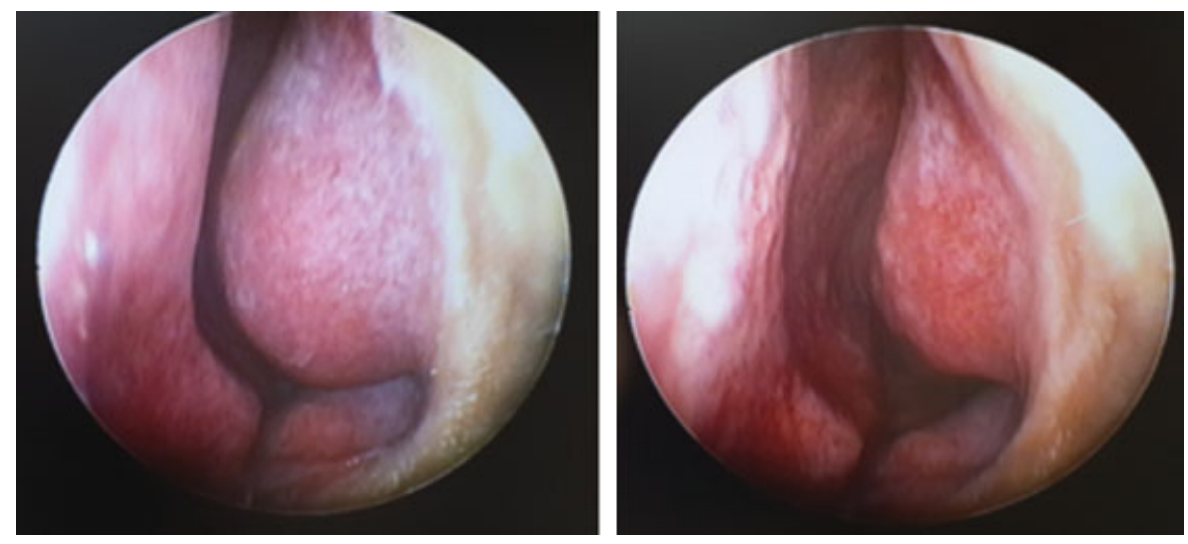

Fig. 2 Left inferior turbinates before and after bipolar electrocautery.

in defense of the upper and lower respiratory tracts. In patients undergoing turbinate reduction, a damaged nasal mucosa and impaired MCC have been implicated in many postoperative side effects such as bleeding, crusting, dryness, defective sense of smell, pain, hyposmia, synechia, and osteonecrosis. ${ }^{5}$ Several methods are available to assess MCC activity. In this study, we used the SCT, which is reliable, simple, and inexpensive. ${ }^{6}$

RFTA is a widely used treatment for nasal obstruction caused by turbinate hypertrophy. The technique is based on local submucosal transport of energy by means of a specifically adapted electrode. The applied energy increases the local temperature of the deep mucosa and causes a thermal lesion without damaging the mucosal surface. The healing process secondarily induces fibrosis and wound contraction, resulting in a reduction in tissue volume. ${ }^{7}$
In BEC, an electrical circuit is established between two probe tips. The resulting thermal energy induces coagulation and thus a reduction in the vascularity and volume of tissue, both in the short and long term. The depth of the burn and lateral thermal injury are less when bipolar rather than unipolar devices are used. ${ }^{8}$

The effects of RFTA on MCC and nasal patency have been investigated in several studies. Uz et al and Parida et al used the SCT and VAS to evaluate the efficacy of RFTA and the postoperative morbidity associated with the procedure..$^{5,9}$ They reported no adverse effects of RFTA on mucociliary activity and a significant improvement in VAS scores. Duran and Ulkü obtained similar results with respect to MCC. ${ }^{10}$ Cukurova et al assessed the long-term (60 months) results of RFTA based on VAS scores and acoustic rhinometry measurements. ${ }^{11}$ The cited authors concluded that 
significant long-term improvements could be achieved with RFTA. Our study confirms these findings.

Taneja and Taneja compared monopolar cauterization, bipolar cauterization, and diathermy, evaluating outcomes with the SCT. ${ }^{12}$ All three techniques led to significant improvements in the quality of life but, based on the SCT values, none yielded significant long-term results. In addition, the authors found that BEC, when performed over the turbinate mucosa, triggered histologic changes involving the transformation of columnar epithelium into cuboidal or squamous epithelium, due to burn injury. The importance of submucosal cauterization while preserving the mucosa was emphasized. ${ }^{12}$ Kilavuz et al assessed RFTA and BEC by VAS, inspiratory peak flowmetry, and magnetic resonance imaging. ${ }^{13}$ They reported significant improvements in the VAS scores and inspiratory peak flowmetry. They noticed that although RFTA and BEC techniques are effective treatment methods, BEC technique is superior to RFTA to achieve high efficiency.

In our patients, the SCT times and VAS scores obtained with the two procedures were comparable and in agreement with results reported in the medical literature. It can therefore be concluded that RFTA and BEC are both effective to improve nasal patency without damaging MCC or causing complications such as bleeding, edema, and crusting. In addition, although cost analysis was not an aim of this study, the economic differences between the two techniques should not be underestimated. BEC equipments are inexpensive and can be used repeatedly, unlike RFTA equipments.

\section{Conclusions}

Submucosal cauterization with preservation of the nasal mucosa and periosteum is an effective, inexpensive, and safe method to achieve a reduction in inferior turbinate hypertrophy.

\section{References}

1 Utley DS, Goode RL, Hakim I. Radiofrequency energy tissue ablation for the treatment of nasal obstruction secondary to turbinate hypertrophy. Laryngoscope 1999;109(5):683-686

2 Cavaliere M, Mottola G, Iemma M. Comparison of the effectiveness and safety of radiofrequency turbinoplasty and traditional surgical technique in treatment of inferior turbinate hypertrophy. Otolaryngol Head Neck Surg 2005;133(6):972-978

3 Sapçı T, Güvenç MG, Evcimik MF. Radiofrequency treatment for inferior turbinate hypertrophy. Kulak Burun Bogaz Ihtis Derg 2011;21(1):56-60

4 Jang YJ, Myong NH, Park KH, Koo TW, Kim HG. Mucociliary transport and histologic characteristics of the mucosa of deviated nasal septum. Arch Otolaryngol Head Neck Surg 2002;128(4):421-424

5 Parida PK, Santhosh K, Ganesan S, Surianarayanan G, Saxena SK. The efficacy of radiofrequency volumetric tissue reduction of hypertrophied inferior turbinate in allergic rhinitis. Indian J Med Sci 2011;65(7):269-277

6 Asai K, Haruna S, Otori N, Yanagi K, Fukami M, Moriyama H. Saccharin test of maxillary sinus mucociliary function after endoscopic sinus surgery. Laryngoscope 2000;110(1):117-122

7 Coste A, Yona L, Blumen M, et al. Radiofrequency is a safe and effective treatment of turbinate hypertrophy. Laryngoscope 2001; 111(5):894-899

8 Massarweh NN, Cosgriff N, Slakey DP. Electrosurgery: history, principles, and current and future uses. J Am Coll Surg 2006; 202(3):520-530

9 Uz AO, Kenar F, Yıldız H, Duran A, Tekin MS, Ayçiçek A. The effect of radiofrequency thermal ablation method on nasal mucociliary activity in patients with inferior turbinate hypertrophy. Kulak Burun Bogaz Ihtis Derg 2014;24(5):247-253

10 Duran M, Ulkü CH. [Effect of radiofrequency thermal ablation treatment on nasal mucociliary clearance in patients with isolated inferior turbinate hypertrophy]. Kulak Burun Bogaz Ihtis Derg 2014;24(4):185-189

11 Cukurova I, Demirhan E, Cetinkaya EA, Yigitbasi OG. Long-term clinical results of radiofrequency tissue volume reduction for inferior turbinate hypertrophy. J Laryngol Otol 2011;125(11):1148-1151

12 Taneja M, Taneja MK. Intra turbinate diathermy cautery V/S high frequency in inferior turbinate hypertrophy. Indian J Otolaryngol Head Neck Surg 2010;62(3):317-321

13 Kilavuz AE, Songu M, Ozkul Y, Ozturkcan S, Katilmis H. Radiofrequency versus electrocautery for inferior turbinate hypertrophy. J Craniofac Surg 2014;25(6):1998-2001 